\title{
Ultrafast all-optical switching with photonic nanojets and semiconductor nanoparticles
}

\author{
Brandon Born, Jeffrey D. A. Krupa, Simon Geoffroy-Gagnon, and Jonathan F. Holzman \\ Integrated Optics Laboratory, The University of British Columbia, School of Engineering \\ 3333 University Way, Kelowna, BC, Canada, V1V 1V7
}

\begin{abstract}
The potential of terabit-per-second fibre optics can be unlocked with emerging all-optical networks and processors employing all-optical switching. To be effective, all-optical switching must support operations with femtojoule switching energies and femtosecond switching times. With this in mind, this work studies geometrical and material characteristics for all-optical switching and develops a new all-optical switching architecture. A nanojet focal geometry is applied, in the form of dielectric spheres, to direct high-intensity photonic nanojets into peripheral semiconductors. Theoretical and experimental analyses demonstrate photonic nanojets, enabling femtojoule switching energies through localized photoinjection, and semiconductor nanoparticles, enabling femtosecond switching times through localized recombination.
\end{abstract}

Keywords: Nanophotonics, nanoparticles, all-optical switching, photonic nanojet, pump-probe, ultrafast.

\section{INTRODUCTION}

Fibre optic networks have the potential for terabit-per-second optical links, however, there is a fundamental limitation in such networks due to the need for far slower gigabit-per-second electronic processing at transmitter/receiver endpoints. Such electronic processors create a bandwidth bottleneck and inhibit the true potential of fibre optics. There is a growing demand to replace these electronic processors with equivalent photonic elements to carry out optical routing, logic, and computing ${ }^{[1],[2]}$. The principle element for this photonic circuitry is the all-optical switch (AOS). All-optical switching is proposed for two implementations: network-on-chip architectures ${ }^{[3]}$, to replace electronic logic with purely all-optical logic, and all-optical multiplexing or demultiplexing ${ }^{[4]}$, to parallelize data into gigabit-per-second streams that are processed by an array of contemporary electronics .

Both of the network-on-chip and multiplexing/demultiplexing architectures require all-optical switching for nonlinear interaction between optical beams. To be sufficiently practical, all-optical switching must consider the demand for low switching energies ${ }^{[5]}$ and ultrafast switching times ${ }^{[6]}$. Miller et al. argue that AOS architectures should target low switching energies, at or below $100 \mathrm{fJ}-$ per-bit ${ }^{[5]}$, while Husko et al. argue that AOS architectures should target ultrafast switching times, at or below $10 \mathrm{ps}^{[6]}$. For low power operation, the $100 \mathrm{fJ}$-per-bit switching energy is suitable, however, a femtosecond (subpicosecond) switching time is still necessary - to allow for integration in terabit-per-second optical fibre networks.

Many AOS technologies have been proposed through the years to address the two aforementioned demands, but it is important to note that these two demands must be met simultaneously to realize a practical AOS architecture. This can be challenging, however, as low switching energies and ultrafast switching times are often mutually exclusive. For example, Lambsdorff et al. ${ }^{[7]}$, Ganikhanov et al. ${ }^{[8]}$, and Gupta et al. ${ }^{[9]}$ focused on lowering the switching time using lowtemperature-grown, ion-implanted, and radiation-damaged GaAs, respectively. Femtosecond switching times were achieved, however the experiments required high beam intensities, or equivalently high switching energies ${ }^{[10]}$. Switching energies can be typically lowered through the use of a resonant AOS architecture. For example, resonant all-optical switching is demonstrated with photonic crystal resonators by Nozaki et al. ${ }^{[11]}$, with ring resonators by Almeida et al. ${ }^{[12]}$ and with quantum dots by Volz et al. ${ }^{[13]}$. In general, resonance builds up the intensity within the device over time to enable a low switching energy, however, this prolongs the switching time. This trade-off is inherent to resonant cavities $^{[14]}$. Practicality issues must also be addressed, especially for all-optical switching with nanometer-scale quantum dots, as coupling is difficult for subwavelength structures.

As seen above, most existing AOS studies have shown that low switching energies and ultrafast switching times are mutually exclusively. There are AOS architectures that have optimized toward both goals, such as the organic photonicbandgap microcavity ${ }^{[15]}$, a top candidate, which achieved a lifetime of 1 ps with a 520 fJ switching energy. However,

Ultrafast Phenomena and Nanophotonics XX, edited by Markus Betz, Abdulhakem Y. Elezzabi, Proc. of SPIE Vol. 9746, 974615 · C 2016 SPIE · CCC code: 0277-786X/16/\$18

doi: $10.1117 / 12.2208636$

Proc. of SPIE Vol. 9746 974615-1 
ultra-sensitive cavities can have difficulties operating with broad spectral inputs, such as those used in wavelength division multiplexing systems.

The goal for this work is to develop a non-resonant AOS architecture that operates with femtojoule switching energies and femtosecond switching times. AOS activation with femtojoule switching energies is achieved through the introduction of a focal geometry, in the form of a dielectric sphere, that can create a high-intensity focal spot, called a photonic nanojet. A semiconductor is placed within the focus of the photonic nanojet, such that the focused beams will photoinject a high charge-carrier density, and thus create a large nonlinearity. AOS activation with femtosecond switching times is achieved through the introduction of a material system, in the form of semiconductor nanoparticles that are embedded in the sphere's surface. The high surface state density in this material system yields rapid chargecarrier recombination - supporting AOS recovery with femtosecond switching times. It is shown that the nanojet focal geometry and nanoparticle material system can be integrated together ${ }^{[16]}$ in an AOS architecture that meets demands for performance and practicality_ particularly for ease of coupling and enhanced stability.

\section{SEMICONDUCTOR CHARGE-CARRIER DYNAMICS}

Charge-carriers induced by an above-bandgap pump beam in a semiconductor can produce a nonlinear perturbation to a below-bandgap probe beam. The dynamics of the charge-carriers that underlie the AOS speed enhancements introduced here. A coupled Drude theory and charge-carrier dynamical model is used to simulate the pump-probe interaction.

The change in transmission of the probe beam, $T$, can be separated into bulk transmission, $T_{\mathrm{b}}$, and surface transmission, $T_{\mathrm{s}}$, according to

$$
\frac{\Delta T(t)}{T}=\frac{\Delta T_{\mathrm{b}}(t)}{T_{\mathrm{b}}}+\frac{\Delta T_{\mathrm{s}}(t)}{T_{\mathrm{s}}}
$$

where the bulk and surface terms have opposing polarities when charge-carriers are introduced into the semiconductor ${ }^{[17]}$. The charge-carrier density, $N(z, t)$, induced by the above-bandgap pump beam creates a differential bulk transmission, $\Delta T_{\mathrm{b}}(t) / T_{\mathrm{b}}$, and a differential surface transmission, $\Delta T_{\mathrm{s}}(t) / T_{\mathrm{s}}$, seen by the below-bandgap probe beam. This is due to $N(z, t)$ increasing the bulk absorption coefficient, $\Delta \alpha_{\mathrm{b}}(t)$, and decreasing the refractive index at the semiconductor's surface, $\Delta n_{\mathrm{s}}(t)$, according to

and

$$
\begin{gathered}
T_{\mathrm{b}}=\mathrm{e}^{-\alpha(t) \delta_{z}}, \\
T_{\mathrm{s}}=\frac{4 n_{s}(t)}{\left(n_{S}(t)+1\right)^{2}},
\end{gathered}
$$

which yield respective bulk and surface transmission differentials of

and

$$
\begin{gathered}
\Delta T_{\mathrm{b}}=-\delta_{\mathrm{z}} \mathrm{e}^{-\alpha(t) \delta_{z}} \Delta \alpha(t), \\
\Delta T_{\mathrm{s}}=-\frac{4\left(n_{s}(t)-1\right)}{\left(n_{s}(t)+1\right)^{3}} \Delta n_{s}(t) .
\end{gathered}
$$

The term $\delta_{z}$ is the photoinjection depth of charge-carriers into the bulk. Substituting Equations (2), (3), (4), and (5) into Equation (1), the overall differential transmission becomes

$$
\frac{\Delta T(t)}{T}=-\delta_{z} \Delta \alpha_{\mathrm{b}}(t)-\frac{n_{\mathrm{s}}-1}{n_{\mathrm{s}}\left(n_{\mathrm{s}}+1\right)} \Delta n_{\mathrm{s}}(t)
$$

where $n_{\mathrm{s}}(t) \approx n_{s}$ is the nominal refractive index near the surface. Both $\Delta \alpha_{\mathrm{b}}(t)$ and $\Delta n_{\mathrm{s}}(t)$ evolve in proportion to the charge-carrier density, $N(z, t)$, with opposing polarities described by

and

$$
\begin{aligned}
& \Delta \alpha(\mathrm{t})=+\frac{e^{2} N(z, t) \Gamma(t)}{c \varepsilon_{0} n_{\mathrm{s}} m(t) \omega^{2}}, \\
& \Delta n_{s}(t)=-\frac{e^{2} N(z, t)}{2 \varepsilon_{0} n_{\mathrm{s}} m(t) \omega^{2}},
\end{aligned}
$$

where $e$ is the fundamental charge, $c$ is the speed of light, $\varepsilon_{0}$ is the permittivity of free space, $m(t)$ is the effective mass, $\omega$ is the angular frequency of the probe, and $\Gamma(t)$ is the scattering rate ${ }^{[17]}$. The scattering rate can also be defined in terms 
of the effective mass, $m(t)$, and the mobility, $\mu(t)$, to be $\Gamma(t)=e /[m(t) \mu(t)]$. Substitution of Equations (7) and (8) into Equation (6) yields

$$
\frac{\Delta T(t)}{T}=\frac{e^{2}}{\varepsilon_{0} n_{\mathrm{s}} \omega^{2}} \frac{N(z, t)}{m(t)}\left[\frac{n_{\mathrm{s}}-1}{2 n_{\mathrm{s}}\left(n_{\mathrm{s}}+1\right)}-\frac{\delta_{z} \Gamma(t)}{c}\right]
$$

The incident pump beam photoinjects charge-carriers into a planar semiconductor. The planar semiconductor is assumed to have a thickness much greater than the photoinjection depth, $\delta_{z}$. The charge-carrier density, $N(z, t)$, evolves through time, $t$, along the dimension, $z$, normal to the surface according to the one-dimensional differential equation

$$
\frac{\partial N(z, t)}{\partial t}=N_{0} \delta(t) \mathrm{e}^{-z / \delta_{z}}-\frac{N(z, t)}{\tau_{\mathrm{b}}}+D \nabla^{2} N(z, t)
$$

The three right-hand side terms in Equation (10) introduce photoinjection, recombination, and diffusion processes, respectively. Photoinjection from an ultrashort pump pulse is represented as the delta-function, $\delta(t)$. The pump beam produces an initial charge-carrier density in the form of an exponential function, decaying from the surface into the semiconductor. The depth of this distribution is defined by the photoinjection depth, $\delta_{z}$, which can be equal to or less than the penetration depth of the given semiconductor. For example, a focal geometry with a short Rayleigh range could produce a $\delta_{z}$ that is less than the penetration depth. The distribution, $N(z, t)$, has an initial charge-carrier density of $N_{0}$ at the surface and is proportional to intensity of the pump beam, given by

$$
N_{0}=\frac{E_{\mathrm{p}} \eta}{\hbar \omega_{\mathrm{p}} V}
$$

The internal quantum efficiency, $\eta$, is typically unity for direct bandgap semiconductors. There is also the reduced Planck's constant, $\hbar=h / 2 \pi$, and angular frequency of the pump beam, $\omega_{\mathrm{p}}$. The photoinjection volume, $V \approx A_{\varnothing} \delta_{z}$, is proportional to the cross-sectional photoinjection area, $A_{\varnothing}$, and photoinjection depth, $\delta_{z}$, of the beam.

The goal is to minimize the switching energy, which is equivalent to minimizing the pump pulse energy, $E_{\mathrm{p}}$, that is required to induce the initial charge-carrier density, $N_{0}$. Femtojoule switching energies can be achieved by decreasing focal spot size, which decreases the photoinjection area, $A_{\varnothing}$, and decreasing the depth of focus, or equivalently photoinjection depth, $\delta_{z}$. In the next section, a nanojet focal geometry is applied to yield a tightly confined focus with a small cross-sectional area and short depth of focus.

The other goal is to minimize the switching time, which is equivalent to minimizing the overall charge-carrier lifetime, $\tau$. This is accomplished by analyzing the boundary condition at the surface of the semiconductor. The boundary condition is linked to Equation (10) and is defined by

$$
\left.D \frac{\partial N(z, t)}{\partial z}\right|_{z=0}=\left.S_{\mathrm{v}} N(z, t)\right|_{z=0}
$$

Due to this boundary condition, charge-carriers rapidly recombine at the semiconductor's surface. Thus, a material system with a sufficient diffusion coefficient, $D$, surface recombination velocity, $S_{\mathrm{v}}$, and a large surface-to-volume ratio, can shorten the overall charge-carrier lifetime, $\tau$, to be far below the bulk charge-carrier lifetime, $\tau_{\mathrm{b}}$. Femtosecond switching times can be achieved by introducing a material system that increases the surface-to-volume ratio, according to the recombination rate relation, $1 / \tau=1 / \tau_{\mathrm{b}}+S_{\mathrm{v}} R$. (This relation is an approximate form for the solution to the Drude/charge-carrier dynamical model presented here.) An increase in the surface-to-volume ratio, $R$, will reduce $\tau$ to be less than $\tau_{\mathrm{b}}$. Prior studies have shown that cylindrical ${ }^{[18]}$ and spherical ${ }^{[19]}$ semiconductor geometries in nanoscale forms exhibit this speed enhancement, as $R$ increases the surface state density.

In summary, the charge-carrier density, $N(z, t)$ can be solved with an inverse Laplace numerical routine, and the differential transmission response, $\Delta T / T$, can then be obtained. The well-known semiconductor GaAs is used as a nominal test. Figure 1(a) depicts the charge-carrier density, $N(z, t)$, as a function of time and depth into a GaAs bulk target, according to Equation (10). The curves for $N(z, t)$ are shown with time increments of $\Delta t=50$ ps. the initial charge-carrier density, $N_{0}$, is calculated via Equation (11) to be $1.4 \times 10^{19} \mathrm{~cm}^{-3}$. Figure 1 (b) depicts the theoretical and experimental (negative) differential transmission responses, $-\Delta T / T$. The experimental differential transmission results are obtained from pump-probe studies of a GaAs bulk target. In this study, the surface recombination velocity, $S_{\mathrm{v}}$, and diffusion coefficient, $D$, are calibrated with the bulk experiment and used for modeling in the next section. 

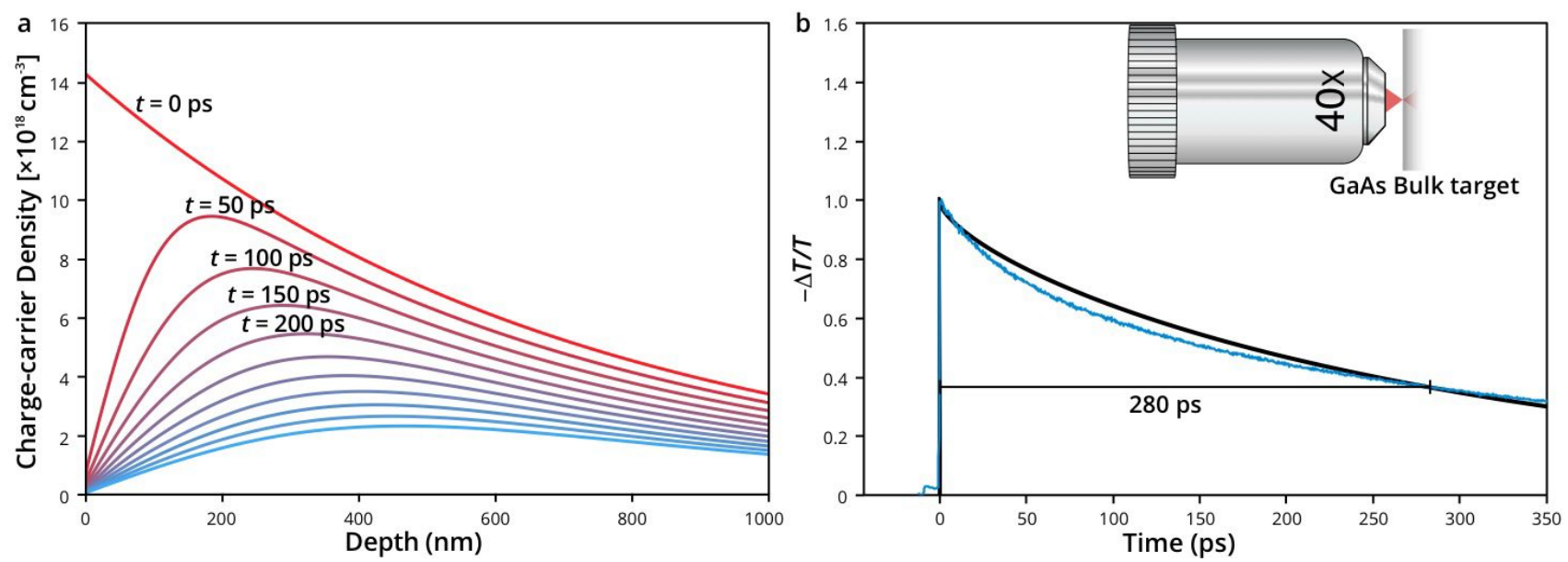

Figure 1. The planar Drude/charge-carrier dynamical model for a GaAs bulk target. (a) The charge carrier density, $\boldsymbol{N}(\mathbf{z}, \boldsymbol{t})$, as a function of time and depth into a GaAs bulk target, according to Equation (10). The curves for $\boldsymbol{N}(\boldsymbol{z}, \boldsymbol{t})$ are shown with time increments of $\Delta \boldsymbol{t}=50$ ps. (b) The differential transmission response, $-\Delta \boldsymbol{T} / \boldsymbol{T}$, theoretical (black) and experimental (blue) curves for a GaAs bulk target. The theoretical response is obtained with Drude theory based on Equation (9) and the time-evolving charge-carrier density. The experimental response is obtained from pump-probe studies using a 40x microscope objective.

\section{PHOTONIC NANOJET FOCAL GEOMETRY}

In an effort to achieve femtojoule switching energies, a focal geometry is introduced to photoinject charge-carriers within a localized volume, defined by the cross-sectional area, $A_{\varnothing}$, and photoinjection depth, $\delta_{z}$. A small charge-carrier volume yields a high initial charge-carrier density, $N_{0}$, in close proximity to the surface of the semiconductor. Thus, it becomes possible to reduce the switching energy and charge-carrier lifetime, $\tau$. In this study, we use a dielectric sphere as the focal geometry with the correct properties to focus light into a high-intensity photonic nanojet ${ }^{[20],[21],[22]}$. Collimated light entering the sphere from one side will form a photonic nanojet just beyond the rear surface of the sphere on the opposing side. The photonic nanojet's tight beam spot and short Raleigh range are well-suited for the necessary localized photoinjection into a semiconductor.

The proposed nanojet based focal geometry is implemented with careful consideration to the properties of the dielectric sphere. There exists a complex interplay between the intensity formed by the photonic nanojet and the dielectric sphere's refractive index, $n$, and diameter, $d$. For this application, seeking a large initial charge-carrier density, $N_{0}$, it is necessary to form the highest possible intensity at the rear surface of the sphere.

Large sphere diameters, defined by the diameter of the sphere being much greater than the wavelength, are considered in this study due to their practical benefits, such as enhanced stability, ease of coupling, and reduced resonance effects. Ray Theory is used to describe this large-scale regime, and to determine the appropriate refractive index that achieves the highest intensity focus. The intensity is estimated by measuring the density of simulated lights rays at the point of interest. The intensity at the rear surface of a sphere intersecting with the optical axis is attained using Ray Theory for varying $n$ and $d>100 \mu \mathrm{m}$. The results are shown in Figure 2. The highest intensities are seen for refractive indices approaching $n \approx 2$. Note that this result can also be seen through a simplification of the thick lens formula ${ }^{[23]}$

$$
f=\frac{n d}{4(n-1)}
$$

for a focal length, $f$, being equal to half the diameter, $d$, when a refractive index of $n=2$ is used. 


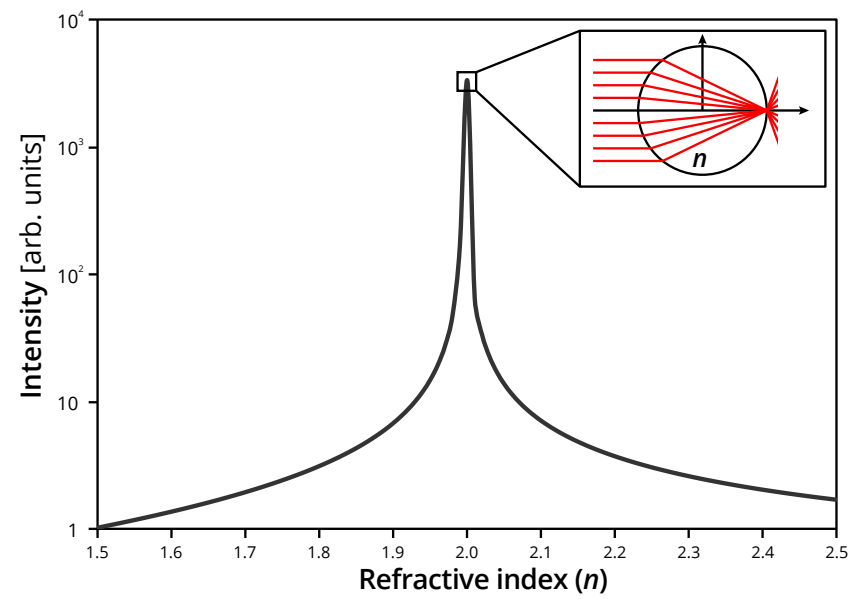

Figure 2. Ray Theory derived intensity on the rear surface of the sphere as a function of refractive index. Based on the maximum ray density, defined as the inverse of the distance between rays, the maximum intensity is found as a function of the refractive index. Ray Theory predicts the intensity to be greatest at a refractive index of $\boldsymbol{n}=2.0$. Ray Theory describes the regime of $\boldsymbol{d}>100 \mu \mathrm{m}$.

To experimentally test the capabilities of the photonic nanojet for localized photoinjection, in terms of switching energy and switching time, 2.00 -mm-diameter spheres of varying refractive indices are studied with a peripheral GaAs wafer placed in the beam's focal spot. A pump-probe differential transmission setup is used for this, as seen in Figure 3, with collimated pump and probe beams. The beams have a $100 \mathrm{fs}$ pulse duration and a repetition rate of $90 \mathrm{MHz}$. For the pump to affect the probe through the photoinjection of charge-carriers, the pump must have an energy above the semiconductor's bandgap and the probe must have an energy below the bandgap — for high transmission and increased sensitivity. The increase in sensitivity is apparent by examining Equations (7) and (8), where the differential transmission is inversely proportional to the angular frequency of the probe squared. The pump and probe wavelengths are $780 \mathrm{~nm}$ and $1550 \mathrm{~nm}$, respectively. The $780 \mathrm{~nm}$ pump beam is near resonance with the band-edge of GaAs. The $1550 \mathrm{~nm}$ probe beam adheres to the telecommunications band, for practical integration with fibre systems. The measurements of the probe beam's change in transmission, $\Delta T / T$, are time-resolved. Samples are acquired as a function of delay between the two pulses using a translational stage and a lock-in amplifier (having a $100 \mathrm{~ms}$ time constant). The experimental setup also has a second harmonic stage, for which the $780 \mathrm{~nm}$ pump beam can be converted to $390 \mathrm{~nm}$ for semiconductors requiring larger energies. The tabletop experimental setup described here characterizes the optical impulse response. (Such a setup is not required for future implementations seeking compact device integration, as time-resolved studies are typically only for material characterization.)

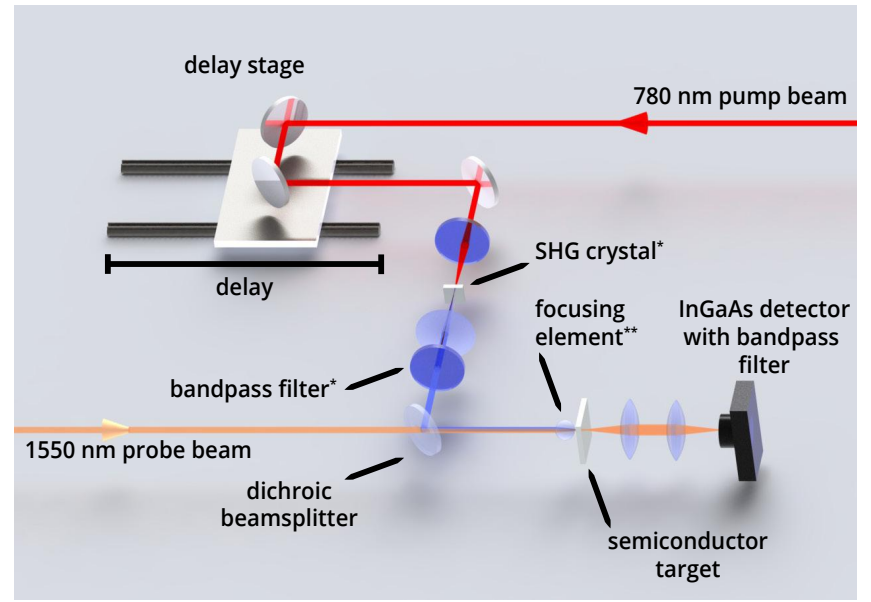

Figure 3. Time-resolved pump-probe spectroscopy experimental setup. A $780 \mathrm{~nm}$ pump beam is delayed using a translational stage. A $1550 \mathrm{~nm}$ probe beam passes through a beamsplitter to become collinear with the pump beam. *A second harmonic generation (SHG) crystal can be introduced to form a $390 \mathrm{~nm}$ pump beam. ${ }^{* *}$ The focusing element is a dielectric sphere, except for the experiment described in Figure 1(b), where it is a microscope objective. 
The experimental results for spheres of varying refractive indices with a peripheral GaAs wafer placed in the beam's focal spot are shown in Figure 4. The differential transmission, $\Delta T / T$, as a function of time, is given for spheres having refractive indices of (a) $n=1.51$, (b) $n=1.76$, (c) $n=1.83$, and (d) $n=1.98$. These refractive indices are quoted for a wavelength of $780 \mathrm{~nm}$, and there is a $n \pm 0.02$ variance for the wavelengths of $390 \mathrm{~nm}$ and $1550 \mathrm{~nm}$. The experimental curves are shown with corresponding theoretical curves, having been generated by the planar Drude/charge-carrier dynamical model. Mie Theory simulations are seen in the figure insets. Experimental (blue curve) and theoretical (black curve) results are given for the probe beam's normalized differential transmission, $\pm \Delta T / T$. The relative strengths of the signals are (a) -0.86 , (b) 0.22 , (c) 0.33 , and (d) 1.0 , which shows that $n=1.98$ has the highest strength or equivalently the lowest switching energy. The negative differential transmission seen for the $n=1.51$ sphere is primarily due to absorption in the bulk, as described by Equation (7), similar to the nominal semiconductor test seen in Figure 1(b) where a microscope objective is used as the focusing element. The increasingly positive differential transmissions seen for the $n=1.76,1.83$, and 1.98 spheres are attributed to increasing contributions from the refractive index at the surface, $\Delta n_{\mathrm{s}}$, as described by Equation (8). This increase is a result of the photoinjected charge-carriers being preferential deposited at the semiconductor's surface.
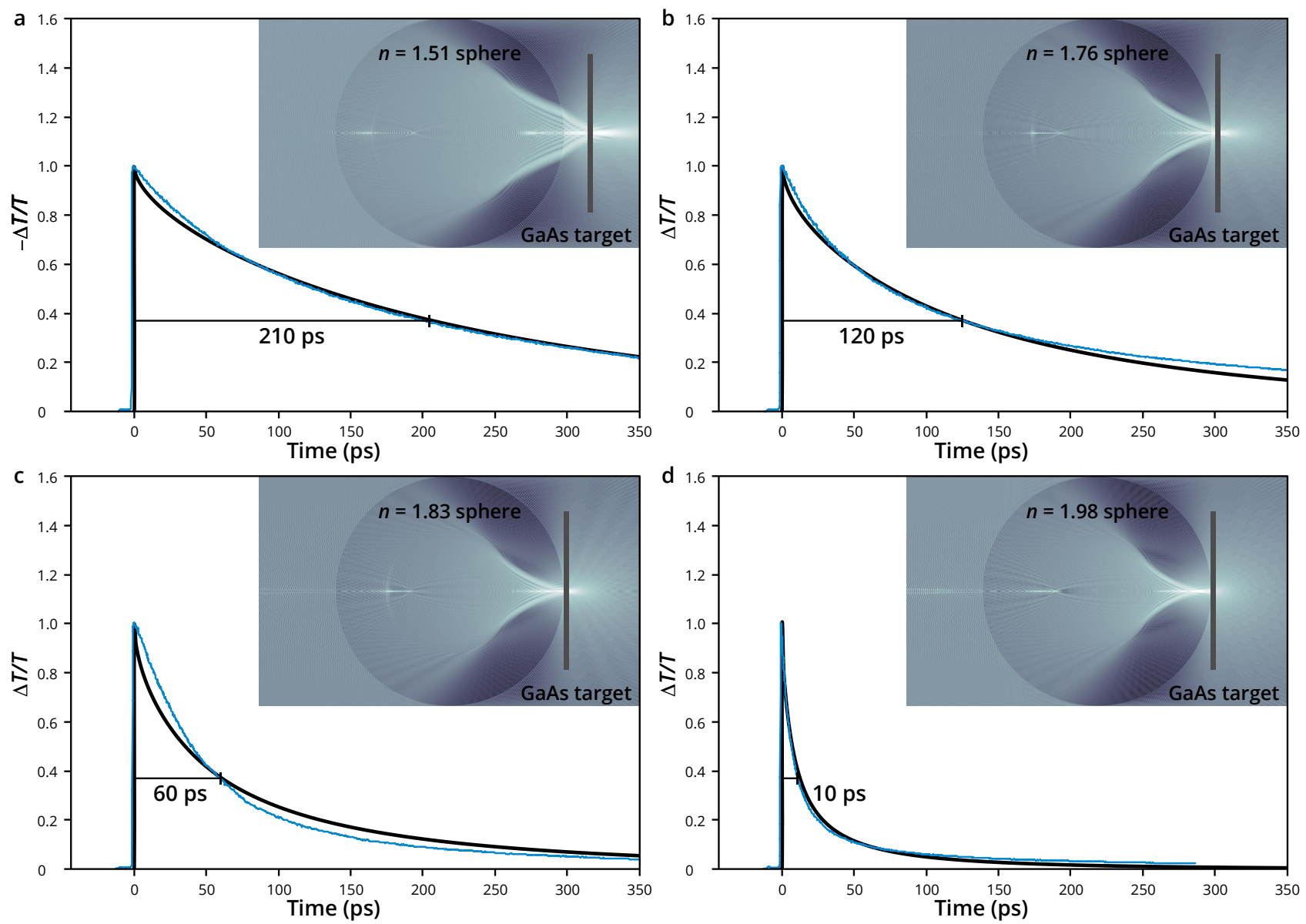

Figure 4. Switching via photoinjection with GaAs using varying sphere refractive indices. Spheres with the refractive indices of (a) $n$ $=1.51$, (b) $n=1.77$, (c) $n=1.85$, and (d) $n=2.00$ are tested. Experimental (blue curve) and theoretical (black curve) results are given for the probe beam's normalized differential transmission, $\pm \Delta \boldsymbol{T} / \boldsymbol{T}$. The relative strengths of the signals are (a) -0.86, (b) 0.22 , (c) 0.33 , and (d) 1.0. The theoretical results are based on the planar Drude/charge-carrier dynamical model. The figure insets are created using Lorenz-Mie Theory simulations with a logarithmic greyscale and are representative of the experiments conducted.

It is worth noting that the increasing (positive) results of Figure 4 exhibit a simultaneous reduction in the chargecarrier lifetime. The charge-carrier lifetime is reduced due to a lower charge-carrier density in the bulk and a higher charge-carrier density at the surface, where there is a large surface recombination velocity, $S_{\mathrm{v}}=1.2 \times 10^{4} \mathrm{~m} / \mathrm{s}$, for GaAs. Spheres with refractive indices of $n=1.51,1.76,1.83$, and 1.98 are measured to have lifetimes of $\tau=210 \mathrm{ps,}$ $120 \mathrm{ps}, 60 \mathrm{ps}$, and $10 \mathrm{ps}$, respectively. The Drude/charge-carrier dynamical model is used to confirm, and the results 
show an increasing (positive) differential transmission and a decreasing lifetime, for a reduction in photoinjection area, $A_{\varnothing}$, and photoinjection depth, $\delta_{z}$, of the pump beam. The Drude/charge-carrier dynamical model predicts the photoinjection depths to be $\delta_{z}=$ (a) 480 , (b) 300 , (c) 180 , and (d) $70 \mathrm{~nm}$, for the experiments.

The minimum switching energy for a sphere, with GaAs at the rear surface and the properties $d=2.00 \mathrm{~mm}$ and $n=$ 1.98 , is estimated to be $10 \mathrm{fJ}$. The minimum switching energy is defined for a signal-to-noise ratio of one in our system. Based on these results, it can be concluded that this AOS architecture can support all-optical switching with femtojoule switching energies and picosecond switching times. The switching time can be further reduced by introducing a material system that promotes localized surface recombination, and this is explored in the next section.

\section{NANOPARTICLE MATERIAL SYSTEMS}

This section investigates localized recombination for ultrafast switching times. Enhanced switching times, or equivalently decreased charge-carrier lifetimes, can be achieve with a semiconductor nanoparticle material system. Semiconductor nanoparticles, with a spherical shape and a nanoscale radius, $a$, have a high surface-to-volume ratio, being $R=3 / a$. This provides more surface area for charge-carriers to recombine and decreases their lifetime, $\tau$, according to $1 / \tau=1 / \tau_{\mathrm{b}}+S_{\mathrm{v}} R$. The nanoparticles are applied to the surface of the $2.00 \mathrm{~mm}$ diameter dielectric sphere with a dry-coating process ${ }^{[24]}$. Such a form facilitates all-optical switching with incident illumination across a full $4 \pi$ steradians solid angle - supporting ease of coupling and parallel operation. Experimental analyses are conducted with the well-known semiconductors $\mathrm{Si}$ and $\mathrm{SiC}$ in nanoparticle form. (Experimental analyses are not conducted with GaAs nanoparticles, due to their high toxicity and limited knowledge in the literature of their safe procedures for laser excitation and handling ${ }^{[25],[26]}$.) The $\mathrm{Si}$ and $\mathrm{SiC}$ nanoparticles tested have radii of approximately $10-50 \mathrm{~nm}$ and $30-60 \mathrm{~nm}$, respectively. As the pump and probe wavelengths are an order of magnitude larger than these nanoparticles, there is negligible scattering witnessed both surfaces where the beam enters and exits.

To experimentally test the capabilities of the nanoparticle material system for localized recombination, in terms of switching energy and switching time, 2.00-mm-diameter spheres with embedded $\mathrm{Si}$ and $\mathrm{SiC}$ nanoparticles are studied with the pump-probe experimental setup. Figure 5(a) and (b) show the differential transmission, $\Delta T / T$, for $\mathrm{Si}$ and $\mathrm{SiC}$ nanoparticles with inset scanning electron microscope (SEM) images and representative Lorenz-Mie Theory simulations depicting the experiments conducted. Based on the bandgap of $\mathrm{Si}$, the experiment uses $780 \mathrm{~nm}$ and $1550 \mathrm{~nm}$ as the respective pump and probe wavelengths. In Figure 5(a), the combined nanojet focal geometry and Si nanoparticle material system is found to have a switching time of $10 \mathrm{ps}$ - which is an improvement (reduction) of approximately two thousand times when compared to bulk $\mathrm{Si}^{[19]}$. The minimum switching energy is estimated to be $200 \mathrm{fJ}$.
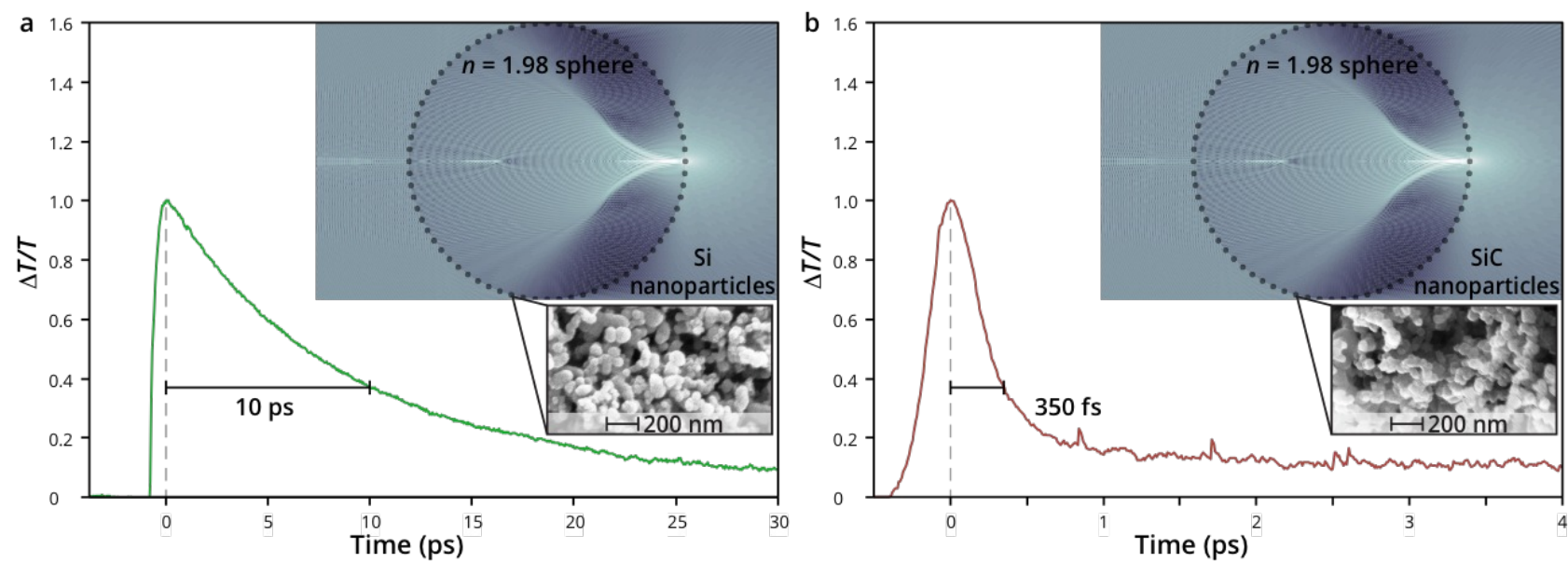

Figure 5. Switching via photoinjection within $\mathrm{Si}$ and $\mathrm{SiC}$ nanoparticles embedded on a dielectric sphere. Experimental (positive) differential transmission for the probe beam, $\Delta \boldsymbol{T} / \boldsymbol{T}$, is shown for (a) Si nanoparticles and (b) SiC nanoparticles. The spheres have a diameter of $\boldsymbol{d}=2.00 \mathrm{~mm}$ and a refractive index of $\boldsymbol{n}=1.98$. Lorenz-Mie Theory simulations are shown, depicting the experiment, in the insets with logarithmic greyscales, along with SEM images of the nanoparticles.

Based on the bandgap of $\mathrm{SiC}$, the experiment uses $390 \mathrm{~nm}$ and $1550 \mathrm{~nm}$ as the respective pump and probe wavelengths. In Figure 5(b), the nanojet focal geometry and $\mathrm{SiC}$ nanoparticle material system is found to have a switching time of 350 fs - which is an improvement (reduction) of approximately sixty million times when compared to bulk $\mathrm{SiC}^{[19]}$. The minimum switching energy is estimated to be $100 \mathrm{fJ}$. It is important to note that the $\mathrm{SiC}$ nanoparticle lifetime exhibits 
two time constants. A longer time constant of 5 ps is observed, which is attributed to the recombination of chargecarriers at the surface of the nanoparticle. This lifetime, although in the picosecond regime, is still a vast improvement over the $22 \mu \mathrm{s}$ bulk lifetime of SiC. In addition, a shorter recovery time constant of $350 \mathrm{fs}$ is observed, which is attributed to ultrafast scattering dynamics in the SiC bandstructure, as photoinjected charge-carriers populate excited states in the M-sidevalley and then undergo intervalley and intravalley scattering ${ }^{[27],[28]}$. The dynamics are seen as a transient effective mass, $m(t)$, and scattering rate, $\Gamma(t)$, through Equations (7) and (8).

Overall, the AOS architecture based on the nanojet focal geometry and $\mathrm{SiC}$ nanoparticle material system meets the demands for femtojoule switching energies and femtosecond switching times-with a $100 \mathrm{fJ}$ switching energy and $350 \mathrm{fs}$ switching time.

\section{CONCLUSIONS}

The geometrical and material characteristics inherent to semiconductor-based all-optical switching were investigated in this work. An AOS architecture was developed, guided by the principle of localization, to achieve all-optical switching with femtojoule switching energies and femtosecond switching times. A focal geometry based upon focusing through a dielectric sphere was used to promote localized photoinjection-by directing high-intensity photonic nanojets into a peripheral semiconductor. Theoretical and experimental analyses were carried out with spheres of differing refractive indices and a GaAs target. It was shown that the nanojet geometry with a sphere refractive index of $n \approx 2$ could localize charge-carriers at the GaAs surface, leading to exceedingly high charge-carrier densities (for a switching energy of $10 \mathrm{fJ}$ ) and surface-assisted charge-carrier recombination (for a $10 \mathrm{ps}$ switching time). A material system based upon nanoparticles was introduced to promote localized recombination-by embedding $20 \mathrm{~nm}$ Si nanoparticles and 50 $\mathrm{nm} \mathrm{SiC} \mathrm{nanoparticles} \mathrm{into} \mathrm{the} \mathrm{sphere} \mathrm{surface.} \mathrm{It} \mathrm{was} \mathrm{shown} \mathrm{that} \mathrm{an} \mathrm{integrated} \mathrm{AOS} \mathrm{architecture,} \mathrm{applying} \mathrm{both} \mathrm{high-}$ intensity photonic nanojets and rapid recombination in nanoparticles, yields all-optical switching with switching energies of $200 \mathrm{fJ}$ and $100 \mathrm{fJ}$, with switching times of $10 \mathrm{ps}$ and $350 \mathrm{fs}$, for the respective $\mathrm{Si}$ and $\mathrm{SiC}$ nanoparticles. These results demonstrate promise for the introduced AOS architecture.

The demonstrated performance levels were brought about by an AOS architecture that supports practical considerations for beam coupling and alignment, as well as physical and temperature stability. Such practical considerations can support the widespread use of this AOS architecture in future devices, with highly complex and dense integration. Future applications may include cascaded implementations accomplished by daisy-chained spheres ${ }^{[29]}$, which is challenging for the majority of AOS devices ${ }^{[30]}$. The omnidirectionality offered by the sphere, allowing for multiple inputs/outputs, could be used to implement parallel implementations ${ }^{[31]}$, or free-space communication systems ${ }^{[32]}$. With the potential of these future applications, the proposed AOS architecture is well-suited for integration with terabit-persecond fibre optic systems.

\section{REFERENCES}

[1] Nazarathy, M. et al. "All-optical linear reconfigurable logic with nonlinear phase erasure," J. Opt. Soc. Am. 26, 21-39 (2009).

[2] Brzozowski, L., \& Sargent, E H. "All-optical analog-to-digital converters, hardlimiters, and logic gates," J. Lightwave Technol. 19(1), 114-119 (2001).

[3] Shacham, A., Bergman, K., \& Carloni, L. P. "On the Design of a Photonic Network-on-Chip," Proceedings of the First International Symposium on Networks-on-Chip. 53-64, (2007).

[4] Ji, H., et al. "Optical Waveform Sampling and Error-Free Demultiplexing of $1.28 \mathrm{~Tb} / \mathrm{s}$ Serial Data in a Nanoengineered Silicon Waveguide," J. Lightwave Technol. 29(4), 426-431 (2011).

[5] Miller, D. A. "Device requirements for optical interconnects to silicon chips," Proc. IEEE 97, 1166-1185 (2009).

[6] Husko, C. et al. "Ultrafast all-optical modulation in GaAs photonic crystal cavities," Appl. Phys. Lett. 94, 021111 (2009).

[7] Lambsdorff, M., Kuhl, J., Rosenzweig, J., Axmann, A. and Schneider, J. "Subpicosecond carrier lifetimes in radiation-damaged GaAs," Appl. Phys. Lett. 58(17), 1881-1883 (1991).

[8] Ganikhanov, F., Lin, G.-R., Chen, W.-C., Chang, C.-Y. \& Pan, C.-L. "Femtosecond carrier lifetimes in arsenicion-implanted GaAs," Appl. Phys. Lett. 67(23), 3465- 3467 (1995).

[9] Gupta, S. et al. "Femtosecond carrier lifetime in GaAs grown by molecular beam epitaxy at low temperatures," Appl. Phys. Lett. 59(25), 3276-3278 (1991).

[10] Elezzabi, A. Y., Meyer, J., Hughes, M. K. Y. \& Johnson, S. R. "Generation of 1-ps infrared pulses at $10.6 \mu \mathrm{m}$ by use of low-temperature-grown GaAs as an optical semiconductor switch," Opt. Lett. 19(12), 898-900 (1994). 
[11] Nozaki, T. et al. "Sub-femtojoule all-optical switching using a photonic-crystal nanocavity," Nature Photon. 4, 477-483 (2010).

[12] Almeida, V. R., Barrios, C. A., Panepucci, R. R. \& Lipson, M. "All-optical control of light on a silicon chip," Nature 431, 1081-1084 (2004).

[13] Volz, T. et al. "Ultrafast all-optical switching by single photons," Nature Photon. 6, 605-609 (2012).

[14] Heuck, M., Kristensen, P. \& Mørk, J. "Energy-bandwidth trade-off in all-optical photonic crystal microcavity switches," Opt. Express 19, 18410-18422 (2011).

[15] Hu, X., Jiang, P., Ding, C., Yang, H. \& Gong, Q. "Picosecond and low-power all-optical switching based on an organic photonic-bandgap microcavity," Nature Photon. 2, 185-189 (2008).

[16] Born, B., Krupa, J. D. A., Geoffroy-Gagnon, S., and Holzman, J. F., "Integration of photonic nanojets and semiconductor nanoparticles for enhanced all-optical switching," Nature Communications, 6, 8097(1-9) (2015).

[17] Collier, C. M., Born, B., Bethune-Waddell, M., Jin, X. \& Holzman, J. F. "Ultrafast photoexcitation and transient mobility of GaP for photoconductive terahertz emission," IEEE J. Quant. Electron. 49, 691-696 (2013).

[18] Holzman, J. F. et al. "Ultrafast carrier dynamics in InP photonic crystals," Nanotechnology. 16, 949-952 (2005).

[19] Collier, C. M., Born, B., \& Holzman, J. F. "Ultrafast response of SiC and Si nanocomposite material systems," Electron. Lett. 48, 1618-1619 (2012).

[20] Chen, Z. G., Taflove, A. \& Backman, V. "Photonic nanojet enhancement of backscattering of light by nanoparticles: a potential novel visible-light ultramicroscopy technique," Opt. Express 12, 1214-1220 (2004).

[21] Li, X., Chen, Z., Taflove, A. \& Backman, V. "Optical analysis of nanoparticles via enhanced backscattering facilitated by 3-D photonic nanojets," Opt. Express 13, 526-533 (2005).

[22] Lecler, S., Takakura, Y., \& Meyrueis, P. "Properties of a three-dimensional photonic jet," Opt. Lett. 30, 2641$2643(2005)$.

[23] Welford, W. T. [Aberration of optical systems], Adam Hilger Ltd. (1986).

[24] Borderieux, S., Wu, C. Y., Bonzongo, J.-C. \& Powers, K. "Control of elemental mercury vapor in combustion systems using Fe2O3 nanoparticles," Aerosol Air Qual. Res. 4, 74-90 (2004).

[25] Flora, S., Dwivedi, N. A "Toxicochemical Review of Gallium Arsenide (Review Paper)," Defence Sci. J. 62(2), 95-104 (2012).

[26] Kuempel, E. D., Castranova, V., Geraci, C. L., Schulte, P. A. "Development of risk-based nanomaterial groups for occupational exposure control," J. Nanopart. Res. 14(9), 1029 (2012).

[27] Ding, J.-L. et al. "Nonlinear optical properties and ultrafast dynamics of undoped and doped bulk SiC," Chin. Phys. Lett. 27, 124202 (2010).

[28] Tomita, T., Saito, S., Suemoto, T., Harima, H. \& Nakashima, S. "Inter-conduction band electron relaxation dynamics in 6H-SiC," Appl. Phys. Lett. 79, 1279 (2001).

[29] Kapitonov, A. M. \& Astratov, V. N. "Observation of nanojet-induced modes with small propagation losses in chains of coupled spherical cavities," Opt. Lett. 32, 409-411 (2007).

[30] Ballarini, D., et al. "All-optical polariton transistor," Nature Commun. 4, 1778 (2013).

[31] Mir, N. F. "An efficient switching fabric for next-generation large-scale computer networking," Comput. Netw. 40(2), 305-315 (2002).

[32] Jin,, X., Hristovski, B. A., Collier, C. M., Geoffroy-Gagnon, S., Born, B., and Holzman, J. F. "Ultrafast all-optical technologies for bi-directional optical wireless communications," Optics Letters, 40, 1583-1586 (2015). 